\title{
Seudoquiste mediastinal y fistulización pleural, excepcional complicación de una pancreatitis
}

\author{
Rodrigo Hernández ${ }^{1}$, Mauricio Pontillo ${ }^{1}$, Eduardo Olivera ${ }^{1}$ y Gustavo Rodríguez ${ }^{1}$
}

\section{Mediastinal pseudocyst and pleural fistulization, exceptional complication of pancreatitis}

Introduction: The thoracic complications secondary to acute pancreatitis are exceptional and even more so the presence of a mediastinal pseudocyst. Case report: 36 year old man. Marijuana and alcohol consumer. History of 6 months of abdominal pain and weight loss of 20 kilos. Installing respiratory symptomatology evolution. Discussion: The forms of presentation of this entity are discussed. Its most frequent etiologies. Emphasis is placed on the role of imaging as well as the analysis of pleural fluid. The therapeutic approach is conservative at the beginning and in some patients it is surgical during evolution; with several options. Key words: Mediastinal pseudocyst; fistulization; pancreatitis.

\section{Resumen}

Introducción: Las complicaciones torácicas secundarias a pancreatitis aguda son excepcionales y más aún la presencia de un pseudoquiste mediastinal. Caso Clínico: Hombre de 36 años. Consumidor de marihuana y alcohol. Historia de 6 meses de dolor abdominal y adelgazamiento de 20 kilos. Instalando en la evolución sintomatología respiratoria. Discusión: Se discuten las formas de presentación de esta entidad. Sus etiologías más frecuentes. Se hace énfasis en el rol de la imagenología así como en el análisis del líquido pleural. El enfoque terapéutico es conservador al inicio y en algunos pacientes es quirúrgico en la evolución; con diversas opciones.

Palabras clave: pseudoquiste mediastinal; fistulización; pancreatitis.

\section{Introducción}

La presencia de seudoquistes mediastinales secundarios a pancreatitis son extremadamente raros $^{1}$.

Las complicaciones torácicas secundarias a pancreatitis son una entidad clínica excepcional (menos del $0,4 \%)^{2}$, presentándose generalmente con derrame pleural y neumonía en pacientes con pancreatitis crónicas recurrentes alcohólicas, también se describe en pancreatitis aguda.

\section{Reporte de caso}

Hombre de 36 años, finlandés. Consumidor de marihuana (42 mg THC/día) y alcohol (60 g/día). Historia 6 meses de evolución de dolor en hemiabdomen superior, adelgazamiento de 20 kilos. En la evolución disnea de esfuerzo y dolor torácico tipo puntada de lado en hemitórax izquierdo. Al examen físico nos encontramos con un paciente con intenso trabajo respiratorio y francamente adelgazado.

TC evidencia extenso derrame pleural bilateral y pseudoquiste en mediastino posterior en relación a pseudoquiste corporocaudal pancreático (Figuras 1, 2 y 3 ). No se evidencian elementos de pancreatitis crónica como calcificaciones, dilatación ductal o atrofia parenquimatosa.

Ecografía abdominal: Vesícula biliar de paredes finas. No se evidencian litiasis. Hepatocolédoco no dilatado. Páncreas aumentado de tamaño con colección de 4 × $3 \mathrm{~cm}$ en su cola. Moderada cantidad de líquido en fondo de saco de Douglas.

Amilasemia inicial: $370 \mathrm{IU} / \mathrm{L}$.

Se realizó drenaje pleural izquierdo mediante pleurostomía. Se obtuvo líquido citrino con alta dosificación de amilasa (6.000 IU/L).
'Clínica Quirúrgica, Hospital Maciel. Montevideo, Uruguay.

Recibido el 28 de julio de 2019 y aceptado para publicación el 16 de septiembre de 2019 .

Correspondencia a: Dr. Rodrigo Hernández rodrih27@hotmail.com 


\section{CASOS CLINICOS}

Figura 1. TC de tórax con contraste endovenoso. Corte axial.
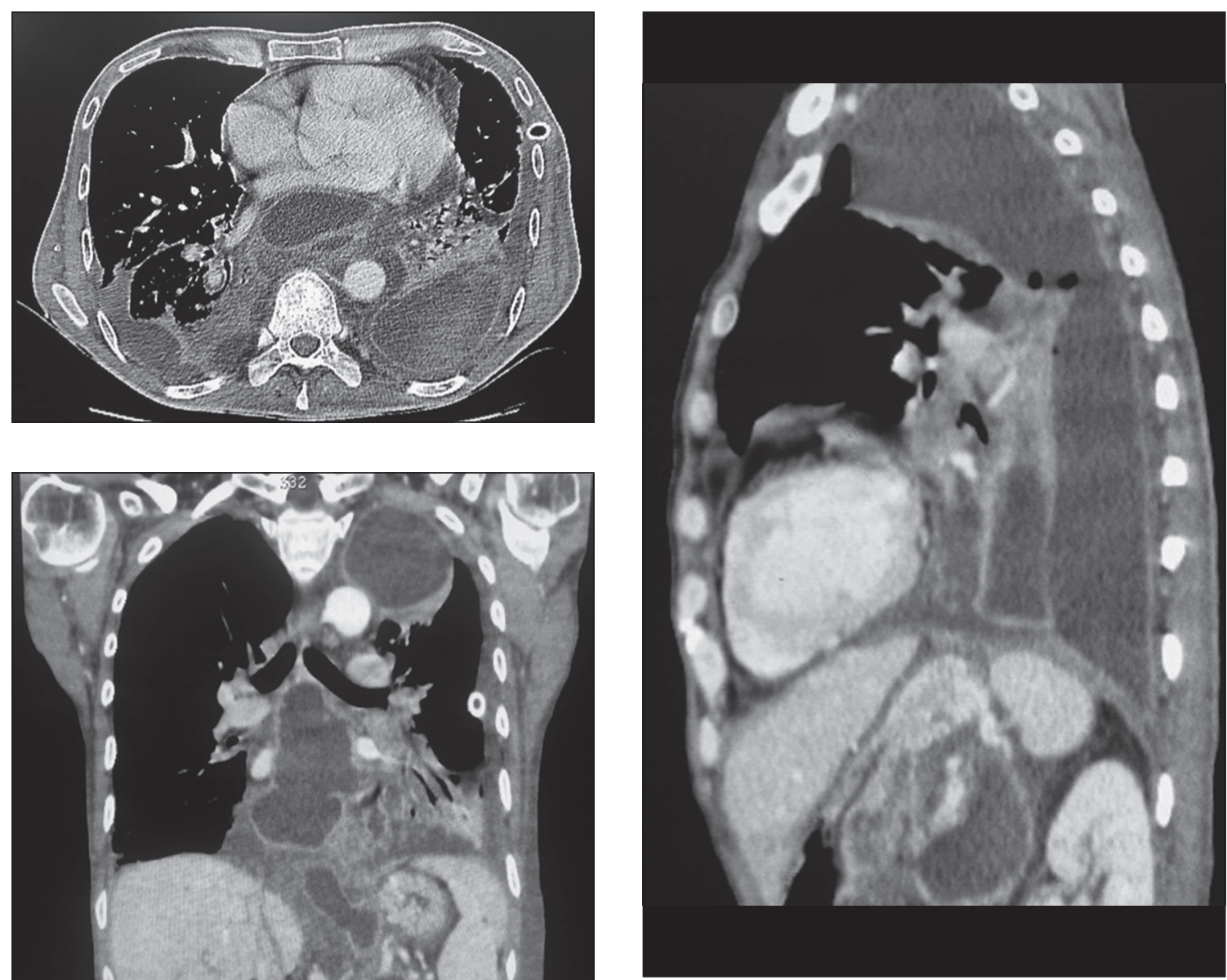

Figura 2. TC de tórax y abdomen con contraste endovenoso. Corte coronal.
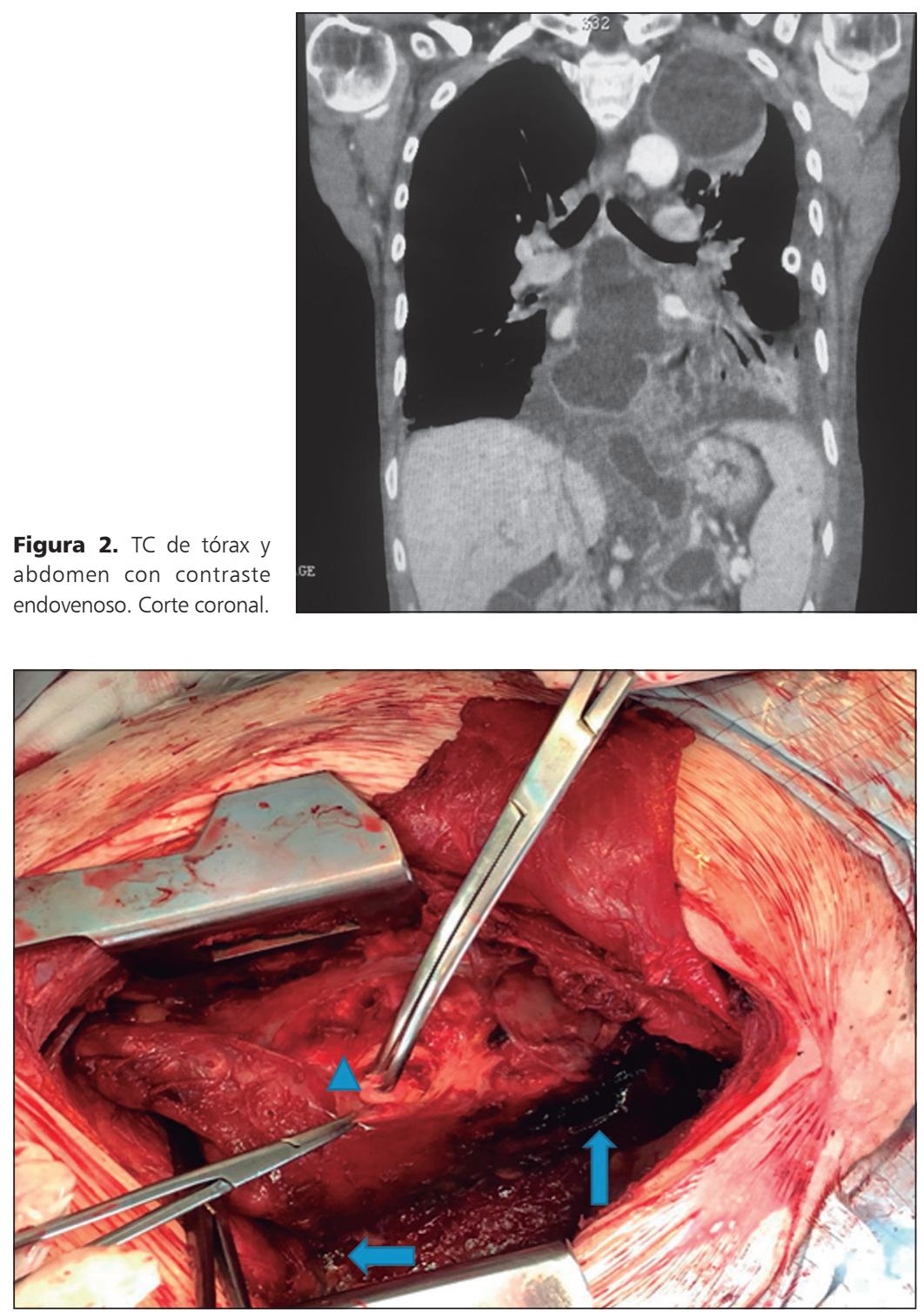

Con diagnóstico de suedoquiste mediastinal secundaria a pancreatitis alcohólica se decidió ingreso a centro de tratamiento intensivo (CTI).

Se inició nutrición parenteral, corrección de sistemas en falla: sobre todo el respiratorio y el hidroelectrolítico.

Si bien el débito de los drenajes se agotó, el paciente persiste con fiebre mantenida e intenso trabajo respiratorio. Apoyados por la TC se decide cirugía.

Se operó a las 2 semanas posteriores al ingreso: abordaje por toracotomía postero lateral izquierda, objetivándose citoesteatonecrosis. Se realiza decorticación pleural y drenaje de colección mediastinal (Figura 4).

Se confecciona yeyunostomía mediante abordaje laparoscópico. De la exploración abdominal si bien se evidencia edema de la fascia de Gerota izquierda no se evidencian colecciones pasibles de drenaje o

Figura 4. Imagen del intraoperatrio donde se muestran la decorticación pleural (punta de desbridamiento. 
Se destaca del cultivo del líquido pleural así como del tejido desbridado el cultivo de Klebsiella pneumoniae ssp y Enterococcus faecalis sensibles a amikacina por lo cual se comienza terapia con dicho antibiótico.

El paciente pasó a sala de cirugía general, permaneciendo sin elementos de SIRS, sin dolor y aumento de peso progresivo.

Requiriendo en total 45 días de internación desde la internación.

\section{Discusión}

Si bien la incidencia de las fístulas pancreatopleurales es incierta, según un estudio realizado por Rockey y Cello revisando la literatura inglesa durante 25 años (1965-1990), la ocurrencia de ésta complicación se daría en menos de $0,4 \%$ de pacientes con pancreatitis ${ }^{5}$.

La fístula se produciría a través del hiato esofágico o aórtico o directamente a través del diafragma ${ }^{3}$. La disrupción posterior del ducto pancreático genera acumulación de líquido pancreático en retroperitoneo, el cual diseca planos atravesando el diafragma a través de sus hiatos determinado las complicaciones torácicas. La disrupción anterior del ducto puede determinar ascitis.

En las pancreatitis crónicas recurrentes se producen pseudoquistes que se comunican y alimentan las fístulas pancreático-torácicas.

Estas fístulas pueden dividirse en 4 tipos:1) Pancreato-pleural; 2) Pancreato-mediastinal (Pseudoquiste mediastinal); 3) Pancreato-bronquial; 4) Pancreato-pericárdica.

La mayoría de estos pacientes se presentan con sintomatología respiratoria inespecífica: disnea, tos, dolor torácico y fiebre. Síntomas que pueden retrasar el diagnóstico y aumenta la morbimortalidad de estos casos

En cuanto a la etiología, la gran mayoría $(84 \%)^{3}$ se trata de pancreatitis crónica alcohólicas y en su mayoría son de sexo masculino $(81 \%)^{3}$, con edad media de presentación de 50 años. Otras etiologías son la pancreatitis aguda y el trauma pancreático.

El análisis del líquido pleural así como la amilasemia son exámenes orientadores al diagnóstico.

La imagenología tiene un papel clave, teniendo la TC una sensibilidad $74 \%$ a $90 \%$ y especificidad de $80 \%$ a $90 \%{ }^{2}$. La colangio resonancia magnética (CRM) tiene una sensibilidad aún mayor.
La misma buscará reconocer la colección mediastinal, mostrando la fístula y el origen pancreático de la misma.

Además de visualizar la anatomía del sistema ductal pancreático lo que es fundamental para efectivizar y dirigir el tratamiento.

El manejo inicial conservador consiste en: nutrición enteral o parenteral, drenaje pleural, estabilización y tratamiento de sistemas en falla. Estas medidas tienen una tasa de éxito de hasta un 30\%-40\% ${ }^{4}$.

El resto requieren tratamiento quirúrgico. En nuestro caso se manejaron las colecciones mediastinales mediante abordaje torácico.

Sabiendo que las modalidades existentes para el drenaje del pseudoquiste son el endoscópico (transgástrico) o el percutáneo. En casos de pancreatitis crónica existen distintas posibilidades que van desde la colangiografía endoscópica con colocación de stent pancreático hasta técnicas más invasivas como la pacreato-yeyunostomía, la pancreatectomía distal.

\section{Conclusiones}

En suma; las fístulas pancreato-torácicas con colecciones mediastinales son una complicación extremadamente rara secundaria a la pancreatitis aguda o crónica.

La dosificación de amilasa en sangre y líquido pleural, así como la imagenología, son claves en su diagnóstico, fundamentalmente la CRM.

El enfoque terapéutico consiste en reposo digestivo y drenaje pleural, de no darse una buena evolución se deberá realizar abordaje quirúrgico.

Sabiendo que existen distintos abordajes y técnicas disponibles, debiendo adecuar a cada caso en particular.

\section{Responsabilidades éticas}

Protección de personas y animales. Los autores declaran que para esta investigación no se han realizado experimentos en seres humanos ni en animales.

Confidencialidad de los datos. Los autores declaran que en este artículo no aparecen datos de pacientes.

Conflictos de interés: no hay. 


\section{CASOS CLINICOS}

\section{Bibliografía}

1. Gupta R, Munoz JC, Garg P, Masri G, Nahman NS Jr, Lambiase LR. Mediastinal pancreatic pseudocyst: a case report and review of the literature. Med Gen Med. 2007;9:8.

2. Tajima Y, Tsutsumi R, Kuroki T, Mishima
T, Adachi T, Kitasato A, et al. Evaluation and management of thoracopancreatic fistula. Surgery 2006;140:773-8.

3. Lew D, Afghani E, Pandol S. Chronic Pancreatitis: Current Status and Challenges for Prevention and Treatment. Digestive Diseases and Sciences 2017;62:1702-12.
4. Choe I. Acute mediastinitis arising from pancreatic mediastinal fistula in recurrent pancreatitis. World Journal of Gastroenterology 2014;20:1499715000.

5. Rockey DC, Cello JP. Pancreaticopleural fistula. Report of 7 patients and review of the literature. Medicine 1990;69:332-44. 